\title{
Semicircular canal dehiscence syndrome
}

INSERM

\section{Source}

INSERM. (1999). Orphanet: an online rare disease and orphan drug data base.

Semicircular canal dehiscence syndrome. ORPHA:420402

Semicircular canal dehiscence (SCD) syndrome is a rare otorhinolaryngologic disease characterized by the uni- or bilateral dehiscence of the bone(s) overlying the superior (most common), lateral or posterior semicircular canal(s). Patients present audiological (autophony, aural fullness, conductive hearing loss, pulsatile tinnitus) and/or vestibular symptoms (sound or pressure-evoked oscillopsia or vertigo, characteristic verticaltorsional eye movements), depending on which semicircular canal is affected. Posterior SCD syndrome is associated with high-riding jugular bulb and fibrous dysplasia, while lateral SCD syndrome is associated with chronic otitis media and cholesteatoma, with or without audiological and vestibular symptoms. 